\title{
Grundrentenplan der großen Koalition
}

\section{Welche Risiken soll die gesetzliche Rentenversicherung versichern?}

\begin{abstract}
Unabhängig davon, ob der Grundrenten-Beschluss der Koalitionsparteien tatsächlich umgesetzt wird, ist er von Interesse, da die Diskussion über die noch offene Finanzierung der geplanten Grundrente auf die grundsätzliche Frage verweist, welche Risiken die Gesetzliche Rentenversicherung eigentlich versichert. Es gibt Argumente dafür, dass die konventionelle Sichtweise auf die versicherten Risiken abgelöst werden könnte bzw. erweitert werden sollte. Das Risiko, trotz langjähriger Erwerbstätigkeit und Beitragszahlung im Alter auf bedarfsgeprüfte Grundsicherung angewiesen zu sein, lässt sich als durch die Rentenversicherung versicherbares Risiko ansehen. Dann ist eine Beitragsfinanzierung nicht nur systematisch möglich, sondern zur Gewährleistung eines verlässlichen Generationsvertrags geradezu geboten.
\end{abstract}

Nicht alle offenen Fragen und Probleme des Beschlusses des Koalitionsausschusses vom 10.11.2019 zur Einführung einer Grundrente ab 2021 können hier diskutiert werden. ${ }^{1}$ Für eine detaillierte Analyse und einige konkreten Umsetzungsvorschläge sei auf das Jahresgutachten 2019 des Sozialbeirats verwiesen, das auch der Ausgangspunkt für die folgenden Überlegungen ist. ${ }^{2}$ Der Sozialbeirat stellt zu Beginn seiner Diskussion des Koalitionsbeschlusses fest: „Die grundsätzliche Einschätzung des Sozialbeirats im Gutachten 2017 (Ziffer 27 ff.) gilt weiterhin: „Es gibt keinen Königsweg für eine Mindestsicherung: Alle bekannten Konzepte konfligieren entweder mit dem Prinzip der Beitragsäquivalenz in der Rentenversicherung, wonach sich die Höhe der Rente nach der Höhe der Beiträge richtet, oder ${ }^{3}$ mit dem Subsidiaritätsprinzip der Grundsicherung, nach welchem zunächst vorhandenes Einkommen einzusetzen ist, bevor (subsidiär) die Solidargemeinschaft Leistungen erbringt." ${ }^{\text {4 }}$

(C) Der/die Autor(en) 2020. Open Access: Dieser Artikel wird unter der Creative Commons Namensnennung 4.0 International Lizenz (https:// creativecommons.org/licenses/by/4.0/deed.de) veröffentlicht.

Open Access wird durch die ZBW - Leibniz-Informationszentrum Wirtschaft gefördert.

* Die Autorin und der Autor geben hier ihre persönliche Meinung und nicht die des Sozialbeirats wieder.

1 Für eine knappe Darstellung des Beschlusses und eine kritische Sicht desselbigen vgl. z.B. W. Schmähl: "Grundrente": Koalitionskitt mit Folgen, in: Wirtschaftsdienst, 99. Jg. (2019), H. 12, S. 814-815.

2 Vgl. Sozialbeirat: Gutachten des Sozialbeirats zum Rentenversicherungsbericht 2019, Berlin, Kapitel III, Ziffern 24 ff.

3 Faktisch konfligieren sie sogar regelmäßig mit beiden Prinzipien gleichzeitig.

4 Vgl. Sozialbeirat, a.a.O., Ziffer 25;. siehe auch ders: Gutachten des Sozialbeirats zum Rentenversicherungsbericht 2016 und zum Alterssicherungsbericht 2016, Ziffer 65; ders.: Gutachten des Sozialbeirats zum Rentenversicherungsbericht 2015, Ziffern 50 ff.
Dann fährt der Sozialbeirat mit einer für inn neuen Überlegung fort:

- „Die Diskussion um eine Grundrente hat aufgezeigt, dass in der Alterssicherungspolitik mindestens drei Gruppen separat betrachtet werden: Einerseits Menschen, die trotz aller individuellen und gesellschaftlichen Bemühungen im Alter oder bei Erwerbsminderung auf Grundsicherung angewiesen sind, zumeist, weil sie nie oder nur sehr kurz überhaupt Beiträge in irgendein Alterssicherungssystem gezahlt haben. Die unzureichenden Alterseinkommen sind in diesen Fällen unstrittig durch die Grundsicherung und nicht durch die Gesetzliche Rentenversicherung auszugleichen. Andererseits gilt für die große Mehrheit der Menschen, dass sie im Alter oder bei Erwerbsminderung absehbar und auch in ihrer Selbsteinschätzung nicht auf Grundsicherung angewiesen oder armutsgefährdet sein werden. Für diese Gruppe ist unstrittig die bestehende

Prof. Dr. Ute Klammer lehrt an der Universität Duisburg-Essen und ist geschäftsführende Direktorin des Instituts Arbeit und Qualifikation (IAQ) sowie Mitglied des Sozialbeirats der Bundesregierung.

Prof. Dr. Dr. h.c. Gert G. Wagner ist Senior Fellow am Deutschen Institut für Wirtschaftsforschung (DIW Berlin) und Mitglied des Sozialbeirats der Bundesregierung. 
Systematik der Alterssicherung, bei allen diskutierbaren Schwächen, dem Grunde nach funktional. Dazwischen liegt die Gruppe, die zunehmend ins Blickfeld gerückt ist: Menschen mit langjähriger sozialversicherungspflichtiger Erwerbstätigkeit, die trotzdem nur geringe Rentenanwartschaften erworben haben. Den Erwerbsbiografien nach ähnelt diese Gruppe mehr der zweiten Gruppe, der großen Mehrheit. Das Armutsrisiko ist für sie aber dennoch insbesondere wegen geringer Löhne, einer Teilzeitbeschäftigung oder nur zum Teil sozialversicherungspflichtigen Erwerbstätigkeit tendenziell erhöht. Für diese Gruppe diskutiert die Politik seit geraumer Zeit Wege, die gewährleisten sollen, dass die Betroffenen im Alter besser stehen, als wenn sie nicht durch ihre langjährige Beitragszahlung zur Rentenversicherung vorgesorgt hätten. Mit anderen Worten: Für die Gruppe, für die trotz langer Versicherungszeiten Rentenansprüche unterhalb des Grundsicherungsniveaus entstehen und damit ein Armutsrisiko nicht ausgeschlossen ist, wird gefragt, ob das Äquivalenzprinzip weiterhin so strikt angewendet werden sollte, wie das rentenrechtlich vorgesehen ist. Die Umsetzung dieses Anliegens ist kontrovers - wie bereits mehrfach vom Sozialbeirat diskutiert wurde."

Und in der Tat: die geplante Grundrente adressiert genau diese Zielgruppe. Nach dem Beschluss des Koalitionsausschusses $^{6}$ ist es Ziel der Grundrente „die Lebensleistung von Menschen anzuerkennen, die jahrzehntelang gearbeitet, Kinder erzogen und Angehörige gepflegt haben“. Zugleich soll sie „einen Beitrag zum Schutz vor Altersarmut leisten“. Nach dem jetzt gewählten Konzept werden beide Zielsetzungen allerdings nur bedingt erreicht: So wird die Lebensleistung der Zielgruppe aufgrund der Einkommensanrechnung nur bei Beziehern geringer Einkommen „anerkannt“. Die gleiche Lebensleistung wird bei unterschiedlichem Haushaltseinkommen durch die Grundrente höchst unterschiedlich bewertet. Faktisch kann ein Mehr an Arbeit und Vorsorge im Ergebnis zu weniger Anerkennung in Form von Grundrente führen. Zugleich wird das allgemeine Ziel des Schutzes vor Altersarmut nicht wirksam angegangen. Dies liegt an der gewählten Zielgruppe von Personen mit einer langjährigen Beitragszahlung - Personen also, die ein unterdurchschnittlich hohes Altersarmutsrisiko aufweisen, während die in Deutschland von Altersarmut Betroffenen mehrheitlich weniger als 35 Jahre an GRV-Versicherungszeiten aufzuweisen haben und insofern nicht von der Grundrente profitieren würden. ${ }^{7}$

5 Sozialbeirat, 2019, a. a. O., Ziffer $26 \mathrm{ff}$.

6 Koalitionsbeschluss zur Grundrente vom 10. November 2019, Teil A, S. 1, https://www.deutsche-rentenversicherung.de/SharedDocs/ Downloads/DE/Presse/191110_koalitionsbeschluss_grundrente. pdf?_blob=publicationFile\&v=1 (14.1.2020).

7 Vgl. Sozialbeirat, 2019, a. a. O., Ziffer 41.
Der Beschluss des Koalitionsausschusses verbindet die Grundrente mit Zielsetzungen, denen sie in großen Teilen nicht gerecht wird. ${ }^{8}$ Entsprechend empfiehlt der Sozialbeirat, „das politische Ziel der jetzt geplanten Grundrente klar zu benennen, nämlich langjährigen Beitragszahlenden zu erleichtern, im Alter eine ausreichend hohe Rente zu beziehen, damit sie ohne Fürsorgeleistung ihren Lebensunterhalt bestreiten können. Die Zielsetzung der politisch gewollten Besserstellung einer bestimmten Versichertengruppe jedenfalls passt zur vereinbarten Konzeption der Grundrente und verbindet sie nicht mit Erwartungen, die mit ihr gar nicht erfüllt werden."9

\section{Wie finanzieren?}

Nach dem Beschluss des Koalitionsausschusses soll die Grundrente aus Steuern und ohne Beitragserhöhung in der Rentenversicherung finanziert werden. Dafür soll der Bundeszuschuss für die Rentenversicherung erhöht werden und als Finanzierungsquelle die im Koalitionsvertrag vereinbarte Finanztransaktionssteuer genutzt werden. Dazu merkt der Sozialbeirat an: „Allerdings ergeben sich trotz der eindeutigen Formulierung Zweifel, ob die verabredete Steuerfinanzierung tatsächlich so umgesetzt wird. Zum einen hat das Bundesarbeitsministerium bereits am Tag nach dem Beschluss des Koalitionsausschusses in einem Erläuterungspapier die getroffenen Vereinbarungen relativiert. Dort heißt es nur noch einschränkend, dass die zur Finanzierung der Grundrente erforderlichen Mittel „insbesondere durch eine Anhebung des Bundeszuschusses aufgebracht werden" sollen, so dass die Grundrente „überwiegend steuerfinanziert“ werde. Des Weiteren ist nach den jahrelangen, bislang ergebnislosen Verhandlungen zur Einführung einer Finanztransaktionssteuer keineswegs sicher, dass diese Steuer beim geplanten Start der Grundrente als Finanzierungsquelle tatsächlich zur Verfügung steht."10

Und weiter heißt es: „Die damit möglicherweise im Lauf des Gesetzgebungsverfahrens aufkommende Frage der Finanzierung der Grundrente steht exemplarisch für eine Diskussion, die seit vielen Jahren bei neuen rentenpolitischen Leistungen geführt wird. Soweit diese Leistungen nicht oder nicht ausschließlich dem Ausgleich versicherungstypischer Risiken dienen bzw. auch an Personen geleistet werden, die dafür keine Beiträge geleistet haben (z.B. vorzeitige abschlagsfreie Altersrente für besonders langjährig Versicherte, zusätzliche Mütterente), wird eine Steuerfinanzierung dieser Maßnahmen gefordert, da es

8 Vgl. C. Fechter et al.: Kompromiss zur Grundrente: Wer profitiert?, in: Wirtschaftsdienst, 99. Jg. (2019), H. 12, S. 843-848, insbesondere S. 848.

9 Vgl. Sozialbeirat, 2019, a. a. O., Ziffer 41.

10 Vgl. ebenda, Ziffer 48. 
sich - je nach Terminologie und Abgrenzung - um „versicherungsfremde", „gesamtgesellschaftliche“ bzw. „nicht beitragsgedeckte Leistungen" handelt."11

Der reflexartige Ruf nach Steuerfinanzierung vereint bei der Einführung entsprechender neuer Leistungen regelmäßig Arbeitgeberseite und Gewerkschaften im gemeinsamen Ziel, einen weiteren Anstieg der Beitragssätze zu verhindern..$^{12} \mathrm{Er}$ ist lauter geworden, seit die Mütterrente eingeführt wurde und aus den Beiträgen der Versicherten finanziert wird. Genauso regelmäßig ist der Ruf nach Steuerfinanzierung aber auch von jenen zu hören, die die Systematik der Gesetzlichen Rentenversicherung (GRV) in Gefahr sehen. Sofern "gesamtgesellschaftliche" Leistungen nicht aus Steuern, sondern aus Beiträgen finanziert werden, führe dies, so die Argumentation, zu einer „Verwässerung“ des Konzepts der einkommensbezogenen und beitragsfinanzierten Gesetzlichen Rentenversicherung. Dies verringere die Rendite der eingezahlten Beiträge - auch im Vergleich zu Formen privater Absicherung - und könne die Akzeptanz der GRV bei den Versicherten schmälern, so z. B. auch die Befürchtung des ehemaligen Vorsitzenden des Sozialbeirats, Winfried Schmähl.13

Ungeachtet einer Reihe sozio-ökonomischer und juristischer Arbeiten ${ }^{14}$ fehlt es allerdings an einer unumstrittenen Definition, welche Leistungen der Rentenversicherung als „versicherungsfremd“, "gesamtgesellschaftlich“ oder „nicht beitragsgedeckt" zu bewerten sind. Folglich lässt sich die Forderung nach Steuerfinanzierung nicht aus bestimmten (Um)Verteilungseffekten ableiten. Dies hat auch der Sozialbeirat in seinem aktuellen Gutachten als Problem markiert: „Der Sozialbeirat sieht es als unbefriedigend an, dass die Frage einer sachgerechten Aufteilung der Finanzierung der Rentenversicherung in Steuer- und Beitragsanteile bis heute ungelöst ist. Die Akzeptanz des Rentenversicherungssystems hängt auch daran, dass die Beitragszahler nicht zur Finanzierung von Aufgaben herangezogen werden, die nicht dem versicherungstypischen Ausgleich dienen. So gehen die Meinungen darüber auseinander, wie Leistungen wie etwa die Grundrente in dieser Hinsicht systematisch einzuordnen sind. Der Sozialbeirat hält es daher für geboten, die Frage

11 Vgl. Sozialbeirat, 2019, a. a. O., Ziffer 49

12 Vgl. aber auch schon B. Rürup: Vom Bundeszuschuss zum Bundesbeitrag in der gesetzlichen Rentenversicherung, in: Deutsche Rentenversicherung, 59. Jg. (2004), H. 10, S. 586-591, S. 586.

13 W. Schmähl, a.a. O., S. 3.

$14 \mathrm{Zu}$ mehreren frühen einschlägigen Arbeiten siehe die vergleichende Rezension von H. Kube: Äquivalenz und Solidarität im Sozialversicherungsrecht, in: Der Staat, 41. Jg. (2002), S. 452-478; und insbesondere A. Wallrabenstein: Versicherung im Sozialstaat, Tübingen 2009, S. 202-233, die die verfassungsrechtlichen Argumente systematisch ordnet. der sachgerechten Finanzierung der Rentenversicherung nach Beitrags- und Steueranteilen grundsätzlich zu klären und nicht weiter diskretionär zu beantworten. “"15

\section{Argumente für eine Beitragsfinanzierung}

Im Folgenden argumentieren wir für eine (mögliche) Beitragsfinanzierung der Grundrente..$^{16}$ Dies stellt jedoch keineswegs die Leitlinie infrage, dass „gesamtgesellschaftliche" Aufgaben über Steuern von der Allgemeinheit getragen werden sollten - zumindest solange, wie nur ein eingeschränkter Ausschnitt der Bevölkerung in der GRV versichert ist und Beiträge entrichtet. ${ }^{17}$ So ist es systematisch richtig, Leistungsausweitungen wie Kindererziehungszeiten respektive Mütterrenten, die der ganzen Bevölkerung offenstehen und niedrigschwellig auch bei Personen zu Leistungsansprüchen führen, die keine weiteren Versicherungszeiten in der GRV haben, ${ }^{18}$ über Steuern zu finanzieren. Hingegen ist die Grundrente bewusst auf langjährig rentenversicherte Personen ausgerichtet und damit eine exklusive Leistung für einen besonders eng und dauerhaft an die GRV gebundenen Personenkreis.

Angesichts der selektiven Zugangsvoraussetzungen zur GRV (man denke an Selbständige, Beamte, über lange Phasen auch geringfügig Beschäftigte, erst recht Nichterwerbstätige) kann hier keineswegs von einer Leistung für die gesamte Gesellschaft gesprochen werden. Ohne Zweifel gibt es gute Gründe dafür, das Risiko unzureichender Alterseinkünfte als einen Tatbestand aufzufassen, der eine "gesamtgesellschaftliche" Aufgabe darstellt und politische Interventionen rechtfertigt. Um dafür eindeutig eine Steuerfinanzierung systemgerecht erscheinen zu lassen, müssten diese Interventionen jedoch auch anderen Gruppen als nur langjährigen GRV-Versicherten zugutekommen.

Dass beitragsfinanzierte Umverteilung innerhalb der Versichertengemeinschaft der Rentenversicherung inhärent ist, zeigt insbesondere diejenige zwischen Gesunden

15 Sozialbeirat, 2019, a. a. O., Ziffer 51.

16 Vgl. für eine stärker sozialrechtliche Sicht G. G. Wagner: Der Grundrenten-Plan der großen Koalition: Praktische und konzeptionelle Probleme - und Möglichkeiten, erscheint in: Die Sozialgerichtsbarkeit, 67. Jg. (2020), H. 2.

17 Die Autorin und der Autor des vorliegenden Beitrags plädieren - wie viele andere - seit Jahren für eine umfassende Versicherungspflicht, vgl. z.B. A. Brettschneider, U. Klammer: Lebenswege in die Altersarmut. Biografische Analysen und sozialpolitische Perspektiven, in: Sozialpolitische Schriften, Bd. 94, Berlin 2016, S. 408-416; G. Rolf, G. G. Wagner: Ziele, Konzept und Detailausgestaltung des „Voll Eigenständigen Systems“ der Altersvorsorge, in: Sozialer Fortschritt, 41 Jg. (1992), H. 12, S. 281-291.

18 So reichen zwei Kinder (sechs Entgeltpunkte) zur Begründung eines - wenn auch niedrigen - Rentenanspruchs in der GRV. Die problematische Teil-Beitragsfinanzierung der Mütterrente soll hier nicht näher diskutiert werden. 
und Erwerbsgeminderten oder zwischen Personen unterschiedlicher sozialer Klassen aufgrund ihrer unterschiedlichen Lebenserwartung ${ }^{19}$ oder zwischen Alleinstehenden und Ehepaaren durch die beitragsfinanzierte Hinterbliebenenversorgung. Diese Umverteilung wird als Risikoausgleich bezeichnet. Es wird hieran also deutlich, dass Umverteilung nicht per se systemfremd im Rahmen einer gesetzlichen Sozialversicherung ist, sondern vielmehr zum Wesensmerkmal einer Sozialversicherung gehört.

Die Hinterbliebenenversorgung zeigt seit 1986 auch, dass eine Versicherungsleistung mit einer Einkommensprüfung verbunden sein kann - wie das bei der Grundrente der Fall sein soll. Dass diese Ausgestaltung der Hinterbliebenenrente durchaus strittig ist, ${ }^{20}$ ändert nichts an der Tatsache, dass diese Ausgestaltung nicht nur real existiert, sondern offenkundig auch verfassungsgemäß ist.

Bei der Grundrente handelt es sich um eine Leistung, die nicht niedrigschwellig der gesamten Bevölkerung offensteht. Sie ist exklusiv auf eine bestimmte Versichertengruppe beschränkt. „Armutsvermeidung“ - als gesamtgesellschaftliche Aufgabe - ist zwar für einen Teil der Berechtigten gewollte Nebenfolge der Grundrente. Doch Armutsvermeidung steht nicht im Vordergrund des Grundrenten-Plans der Koalition.

Bei der Grundrente handelt es sich nach persönlicher Überzeugung der Autorin und des Autors dieses Beitrags um eine Versicherung gegen das Risiko, trotz langjähriger Mitgliedschaft und Beitragszahlung keine armutsvermeidenden Alterssicherungsansprüche aufbauen zu können. ${ }^{21}$ Dies ist - neben der Versicherung gegen Langlebigkeit, gegen Erwerbsminderung und gegen den Tod mit Hinterlassung Hinterbliebener - ein viertes Risiko, das die GRV zukünftig für die Gruppe der Versicherten abdecken kann und sollte und ist insoweit keineswegs eine „versicherungs-

19 Vgl. F. Breyer, S. Hupfeld: Neue Rentenformel - mehr Gerechtigkeit und weniger Altersarmut, in: DIW-Wochenbericht, Nr. 5, 2009, S. 82-86.

20 So kritisiert Winfried Schmähl „Seit 1986 werden Hinterbliebenenrenten [ ...] in voller Höhe [...] nur noch dann gezahlt, wenn eine eigene Versichertenrente sowie Arbeitsentgelt des überlebenden Ehegatten unterhalb eines Freibetrags bleiben. [...] Eine solche bedarfsorientierte Leistung ist [...] sachgerecht aus allgemeinen Haushaltsmitteln und nicht aus am Arbeitsverhältnis anknüpfenden Sozialversicherungsbeiträgen zu finanzieren." Schmähl fügt hier die aufschlussreiche Fußnote an „Diese Auffassung wird insbesondere von Vertretern der Rentenversicherungsträger abgelehnt.“ W. Schmähl: Finanzpolitik und Rentenversicherung - Beispiele für die Notwendigkeit einer integrierten Sichtweise, in: H. P. Galler, G. G. Wagner (Hrsg.): Empirische Forschung und wirtschaftspolitische Beratung - Festschrift für HansJürgen Krupp zum 65. Geburtstag, Frankfurt und New York 1998, S. 448-468. Auch B. Rürup, a. a. O., S. 586 zählt „Teile der Hinterbliebenenversorgung" zu den „nicht beitragsgedeckten Leistungen“.

21 Beispielsweise sieht auch der liberale Ökonom Friedrich Breyer „eine Reduktion des Risikos für Altersarmut unter langjährig Versicherten“ als sinnvolles Ziel an, F. Breyer, S. Hupfeld, a.a. O., S. 86. fremde Leistung“. ${ }^{22}$ Durch die mit der Rentenreform 2001 eingeleitete Absenkung des Rentenniveaus ist das Risiko, trotz langjähriger Systemzugehörigkeit und Beitragszahlung keinen Rentenanspruch oberhalb des Grundsicherungsniveaus zu erreichen, zunehmend gestiegen und wird in Zukunft weiter steigen. Erreichte ein Durchschnittsverdiener 2009 bereits nach 26,2 Jahren einen Rentenanspruch auf Grundsicherungsniveau, wird er 2045 hierfür nach geltenem Recht perspektivisch 33,4 Jahre benötigen. Wer aufgrund von Teilzeitarbeit oder auch Niedriglöhnen nur die Hälfte des Durchschnittseinkommens verdient, benötigte schon 2009 52,4 Erwerbs- und Beitragsjahre um mit seinem Rentenanspruch mit dem Regelbedarf für einen alleinstehenden Grundsicherungsempfänger (inklusive Kosten der Unterkunft) gleichzuziehen (vgl. Abbildung 1).

Angesichts der Kombination aus der Absenkung des Rentenniveaus mit der Zunahme von Teilzeitarbeit und Niedriglöhnen kann das Sicherungsversprechen der GRV, vor Armut im Alter zu schützen, für einen steigenden Teil der Versicherten insofern nicht mehr eingelöst werden. Es ist daher die Frage zu stellen, ob die Legitimität und Akzeptanz der Rentenversicherung nicht stärker dadurch gefährdet ist, dass Menschen ihr Leben lang arbeiten, ordnungsgemäß einzahlen und dennoch nicht mehr erhalten als andere, die nie Beiträge entrichtet haben, als es der Fall sein wird, wenn innerhalb der Solidargemeinschaft GRV bei Wahrung der Teilhabeäquivalenz eine relative Besserstellung von Personen mit schwachen Erwerbsbiografien zulasten der besser verdienenden Beitragszahler stattfinden würde.

Bei der Entscheidung für mehr Umverteilung zugunsten langjährig Versicherter sollte bedacht werden, was eigentlich das Ziel der ungleichen Leistungshöhe innerhalb der Rentenversicherung ist. Wie die Juristin Astrid Wallrabenstein sehen wir es als eine zentrale Aufgabe der ungleichen Rentenzahlungen bzw. der Beitrags-Leistungs-Äquivalenz an, „dass die Beitragslast von den Betroffenen akzeptiert wird." ${ }^{23}$ Und dabei kommt es „in keiner Weise auf eine mathematisch exakte Äquivalenz der Leistung im Verhältnis zur Beitragshöhe an“24 - diese lässt

22 Es ist auch keineswegs theoretisch und empirisch hinreichend untersucht, sondern wird in der Regel bloß behauptet, dass auf einen „Versicherungskern“ reduzierte Sozialversicherungen stärker akzeptiert würden als „umverteilende“ Sozialversicherungen, vgl. schon G. G. Wagner: Perspektiven der Alterssicherung, in: R. Hauser (Hrsg.): Die Zukunft des Sozialstaats, Berlin 2000 (zugleich auch Beiheft 8 der Zeitschrift für Wirtschafts- und Sozialwissenschaften), S. 133 und die dort zitierte Literatur.

23 A. Wallrabenstein: Äquivalenz in der Sozialversicherung - aber am richtigen Ort, in: W. Durner et al. (Hrsg.): Das sinnvoll Denkbare denken, das davon Machbare machen - Gedächtnisschrift für Arndt Schmehl, Berlin 2019, S. S. 312, auch mit Bezug auf Bryde und Schmehl.

24 Ebenda, S. 312. 
Abbildung 1

Überschreitung von Grundsicherungsbedarf und Rente bei sinkendem Rentenniveau

nach Entgeltposition und Beitragsjahren, 2009 bis 2045

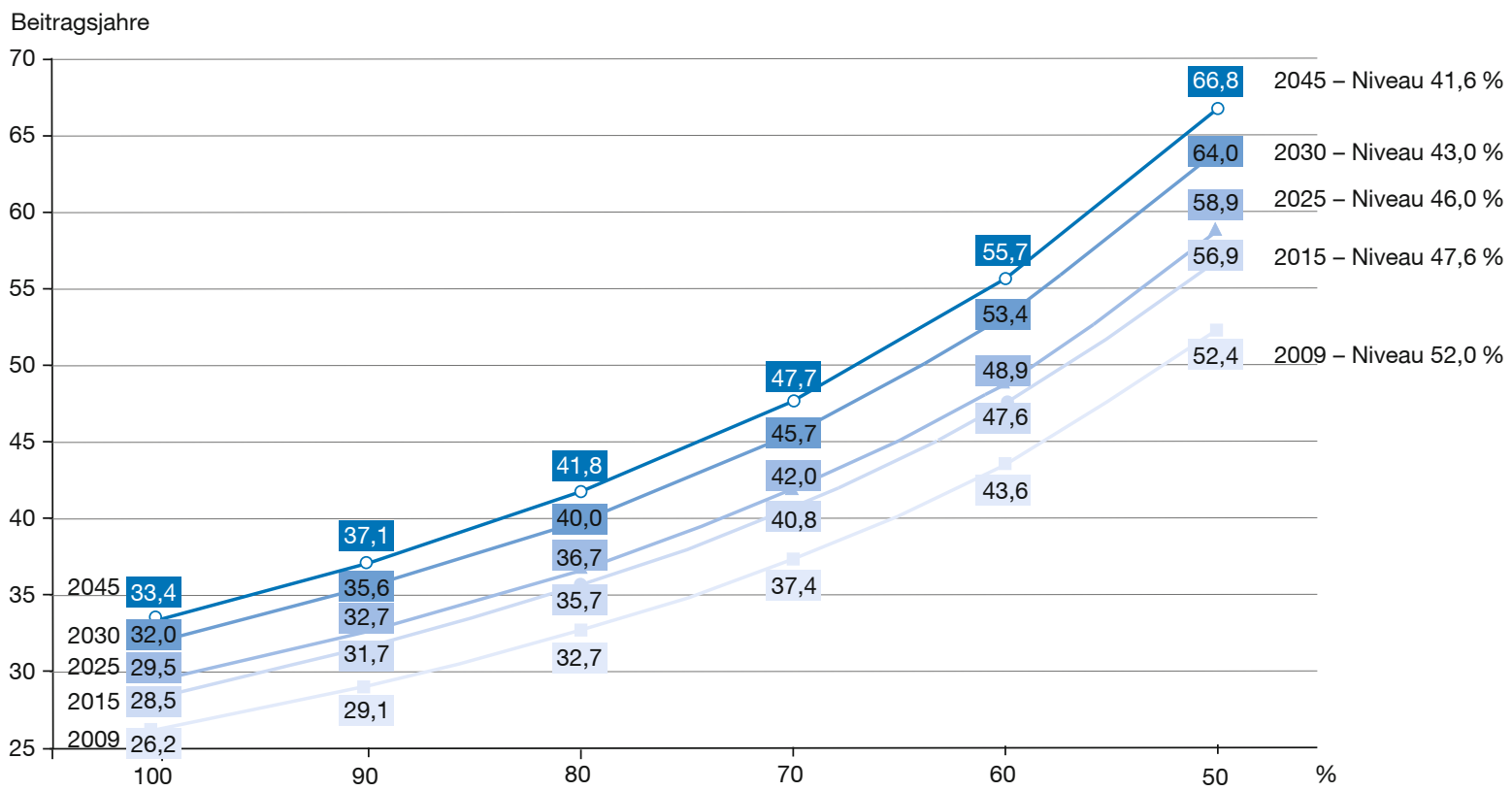

Anmerkungen: Annahmen (im Detail siehe Kommentierung): Grundsicherung im Alter: Bedarf für Alleinstehende (Regelbedarf und bundesdurchschnittliche Kosten der Unterkunft) im Jahr 2015: 747 Euro. Rente: Nettorente vor Steuern, Beitragsabzüge (Krankenversicheurng und Pflegeversicherung der Rentner) Werte für 2015. Niveau 2009, 2015 (Deutsche Rentenversicherung), 2025 (Rentenversicherungsbericht, 2030 (Niveausicherungsklausel), 2045 (Bundesregierung).

Quelle: Institut Arbeit und Qualifikation (IAQ), www.sozialpolitik-aktuell.de; eigene Berechungen.

sich angesichts vieler relevanter Variablen, zumal im Lebensverlauf, ohnehin kaum objektiv und insbesondere ex ante feststellen. ${ }^{25}$ Relevant ist vielmehr die Einschätzung der Fairness sozialstaatlicher Leistungen - im Vergleich zu anderen Versicherten, aber eben auch im Vergleich zu Nichtversicherten.

Betrachtet man zudem das Risiko, trotz langjähriger Erwerbstätigkeit keine armutsvermeidenden Rentenansprüche aufbauen zu können, als ein Risiko, das ex ante jeden Versicherten treffen kann, so stellt seine Absicherung keine „Umverteilung“, sondern einen Risikoausgleich zwischen Versicherten dar, der für viele staatliche Rentensysteme der ersten Säule in Europa und darüber hinaus durchaus konstitutiv ist. ${ }^{26}$

Mit anderen Worten: Durch die Grundrente - wenn sie Gesetz werden sollte - könnte die Legitimation der Rentenversicherung in der Bevölkerung insofern gestärkt

25 Vgl. z.B. G. G. Wagner: Umverteilung in der gesetzlichen Rentenversicherung - Eine theoretische und empirische Analyse zum Versicherungsprinzip in der gesetzlichen Rentenversicherung, Frankfurt und New York 1984.

26 Beispielhaft genannt seien die Rentensysteme der Schweiz, Österreichs oder der Niederlande. werden, weil bestimmte Folgen der Deregulierung des Arbeitsmarkts, die zu niedrigen Löhnen und unstetigeren Erwerbsverläufen führen, das Sicherungsversprechen der Gesetzlichen Rentenversicherung nicht mehr infrage stellen würden. Eine verlässliche Grundrente nach langjähriger Beitragszahlung würde dieses Risiko im Rentenalter kompensieren. ${ }^{27}$

Zudem dürfte eine beitragsfinanzierte Umverteilung (oder Risikoausgleich) polit-ökonomisch stabiler sein als ein steuerfinanziertes Regime. ${ }^{28}$ Denn der Gesetzgeber hat

27 Der Mitautor dieses Beitrags gesteht, dass er dieses spezielle Risiko erst in den letzten Jahren als solches erkannt hat. In einer umfassenden Übersicht über Gefahren, die für die Versicherungs-Vorsorge geeignet sind (vgl. G. G. Wagner: Zentrale Aufgaben beim Um- und Ausbau der Gefahrenvorsorge, in: J. Genosko (Hrsg.): Reform des Sozialstaats II, Berlin 1998, S. 26) wird das Risiko von Armut- und Armutsnähe trotz langjähriger Erwerbstätigkeit nicht genannt, sondern der Ausgleich von „Pech“ wird der Fürsorge zugewiesen. Vgl. auch T. Köhler-Rama: Grundrente ist kein Systembruch - eine Replik, in: Wirtschaftsdienst, 99. Jg. (2019), H. 6, S. 433 ff.; und für eine Fülle von Gegenargumenten F. Ruland: Vorschlag zur Grundrente: ungerecht, ineffizient und teuer, in: Wirtschaftsdienst, 99. Jg. (2019), H. 3, S. 189195; und F. Ruland: Es bleibt dabei: Die Grundrente taugt nichts - eine Erwiderung, in: Wirtschaftsdienst, 99. Jg. (2019), H. 6, S. 436-439.

28 Vgl. z.B. G. G. Wagner: Verlässlichkeit einer höheren Steuerfinanzierung der sozialen Sicherung ist die zentrale Frage, in: Wirtschaftsdienst, 85. Jg. (2005), H. 5, S. 283-287. 
bei der Reform eines beitragsfinanzierten Systems größere politische Widerstände zu überwinden als bei einem steuerfinanzierten System, ${ }^{29}$ das intertemporal weniger Verlässlichkeit bietet. Schließlich ist auch zu bedenken, dass unerwünschte Verteilungswirkungen einer Beitragsfinanzierung umso geringer werden, je vollständiger die Versicherungspflicht für die Gesetzliche Rentenversicherung alle Erwerbstätigen umfasst. ${ }^{30}$ Mit der von der Bundesregierung konkret geplanten Versicherungspflicht für Selbständige wird hier eine wesentliche Lücke geschlossen werden.

Nach unserer persönlichen Überzeugung kann auch den Arbeitgebern über ihre Beiträge zur Rentenversicherung zugemutet werden, für die Absicherung des Grundrentenrisikos aufzukommen. Denn die Deregulierung des Arbeitsmarktes hat nicht nur Vorteile, nämlich mehr Arbeitsplätze zu ermöglichen, sondern sie hat auch ihren Preis. Viele Erwerbstätige wissen nicht, ob sie im Alter eine auskömmliche Rente haben werden oder von Armut bedroht sind. Diese Unsicherheit zu verkleinern, gehört zu einer vernünftigen Gesellschaftspolitik. Diese nützt auch den Arbeitgebern, die von der Deregulierung profitiert haben. Beiträge in die GRV, die zur Milderung der sozialen Folgen der Deregulierung beitragen, entlasten zugleich bei den Steuern, da angesichts des grundgesetzlich verbrieften Sozialstaatsgebots ansonsten über Steuern zusätzliche Grundsicherungsleistungen zu finanzieren wären. Im Übrigen können Arbeitgeber diese Finanzierungslast verkleinern, indem sie Arbeitsplätze und Löhne anbieten, die im Alter eine Grundrente überflüssig machen. Insofern ist eine beitragsfinanzierte Grundrente im Jargon der Ökonomen - anreizkompatibel.

\section{Fazit}

Das von Bundesarbeitsminister Heil vorgelegte Grundrentenkonzept ist komplex und in Einzelheiten sowie bezüglich bestimmter Verteilungswirkungen zweifellos kritisierbar. ${ }^{31}$ Es adressiert aber auf jeden Fall eine Frage, die angesichts veränderter demografischer und arbeitsmarktpolitischer Rahmenbedingungen in Kombination mit absehbaren weiteren Leistungseinschränkungen bei den Rentenzahlungen dringend thematisiert und diskutiert werden muss: Die Frage nach dem „Sicherungsversprechen“ der Gesetzlichen Rentenversicherung. Vieles spricht für die Hypothese, dass die Akzeptanz der GRV und die Bereitschaft zur Beitragszahlung (weiter) sinken würde, wenn Versicherte trotz langjähriger Beitragszahlung zunehmend keinen Rentenanspruch oberhalb des allgemeinen Grundsicherungsniveaus mehr erreichen würden.

Die Garantie eines Leistungsanspruchs für langjährig Versicherte, der erkennbar oberhalb der allen Staatsbürgern zustehenden Grundsicherung liegt, wäre ein Beitrag zur Stärkung der Verlässlichkeit des Rentensystems und damit völlig kompatibel mit den Aufgaben einer Sozialversicherung im Rahmen eines verlässlichen Generationsvertrags. Betrachtet man den Ausgleich bestimmter Erwerbs- und Einkommensrisiken zwischen den GRVMitgliedern als versichertes Risiko, so wäre die Finanzierung über Beiträge (und nicht über Steuern) folgerichtig und sachgerecht.

Title: Basic Pension Plan of the Grand Coalition

Abstract: Irrespective of whether the basic pension resolution of the coalition parties is actually implemented, it is relevant because the ongoing discussion about the financing of the planned basic pension refers to the fundamental question about risks that the Statutory Pension Insurance actually insures. There are arguments that the conventional view of the insured risks could or should be replaced. The risk of being dependent on means-tested basic provisions despite many years of employment and paid contributions could be regarded as a risk insurable by the pension insurance. In this case, financing contributions would not only be systematically possible, but virtually imperative.

JEL Classification: D31, D63, H55 